\title{
Reconstruction of large tibial bone defects following osteosarcoma resection using bone transport distraction: A report of two cases
}

\author{
ZHENGMING YANG, LIBIN JIN, HUIMIN TAO and DISHENG YANG \\ Department of Orthopaedics, Second Affiliated Hospital, School of Medicine, \\ Zhejiang University, Hangzhou, Zhejiang 310009, P.R. China
}

Received April 1,2015; Accepted June 7, 2016

DOI: $10.3892 / \mathrm{ol} .2016 .4777$

\begin{abstract}
The clinical efficiency of bone transport distraction osteogenesis in the reconstruction of large tibial defects following resection of osteosarcoma remains unclear. The current study presents two cases of large tibial defects treated with bone transport distraction using an Orthofix external fixator. Case 1 was a 29-year-old man with a tibial defect $11 \mathrm{~cm}$ in length, while case 2 was a 16 -year-old girl with a 15 -cm-long defect. Bone transport distraction osteogenesis was initiated for the both cases on day 14 following resection of the tibial osteosarcoma. Bone transport distraction in case 1 and 2 was continued for 16 and 28 months, respectively, and the patients were followed up for 51 and 56 months, respectively. The two patients did not exhibit any signs of tumor recurrence or tumor metastasis during the follow-up period. The Musculoskeletal Tumor Society functional scores at final follow-up visits were 22 and 18 for case 1 and 2, respectively. Based on the experience gained in these 2 cases, a bone transport is a viable option for the reconstruction of large tibial defects following osteosarcoma resection.
\end{abstract}

\section{Introduction}

Over the past few years, the five-year survival rate of patients with osteosarcoma has remarkably improved with the widespread use of neoadjuvant chemotherapy, and limb salvage surgery is now the mainstay in the treatment of this malignancy (1-3). Currently, several options are available for limb reconstruction following the resection of malignant tumors, including tumor prostheses, allografts, and vascularized, autologous osseous grafts. However, reconstruction of large tibial bone defects resulting from the resection of osteosarcomas remains challenging (4-6). Bone transport distraction

Correspondence to: Dr Libin Jin, Department of Orthopaedics, Second Affiliated Hospital, School of Medicine, Zhejiang University, 88 Jiefang Road, Hangzhou, Zhejiang 310009, P.R. China E-mail: tongxun2011@sohu.com

Key words: bone tumor, distraction osteogenesis, skeletal reconstruction osteogenesis is widely used in the reconstruction of large bone defects following trauma, but its use has rarely been reported in defects resulting from the removal of bone tumors (7). This is primarily due to concerns regarding the possible detrimental effects of chemotherapy administered for osteosarcoma on bone transport osteogenesis and bone union.

The current case report presents two cases in which the bone transport technique was successfully used for the reconstruction of large tibial bone defects caused by the resection of osteosarcomas. Written informed consent was obtained from each patient for publication of this study.

\section{Case report}

Case 1. A 29-year-old man was admitted to the Second Affiliated Hospital (Hangzhou, Zhejiang, China) in April 2009 due to pain in the right proximal tibial region, which had been ongoing for 4 months. Biopsy of the tissue sample collected from the affected site revealed that the lesion was a parosteal osteosarcoma. Subsequently, the lesion was surgically resected. No evidence of metastasis was detected during the surgery. The tibial defect was $11 \mathrm{~cm}$ in length and fixed with a Orthofix Limb Reconstruction System via an external fixator (Orthofix, Lewisville, TX, USA) (Fig. 1). Post-surgery, the patient was administered with two cycles of chemotherapy. Each cycle consisted of methotrexate $\left(8 \mathrm{~g} / \mathrm{m}^{2}\right)$ on days 1 and 8 , cisplatin $\left(120 \mathrm{mg} / \mathrm{m}^{2}\right)$ on day 15 and doxorubicin $\left(60 \mathrm{mg} / \mathrm{m}^{2}\right)$ on day 17 . The second cycle of chemotherapy was started after 2 weeks. Bone transport distraction osteogenesis was initiated on postoperative day 14 at a distraction rate of $2.0 \times 0.5 \mathrm{~mm}$ per day. The callus to diameter ratio (CDR) was calculated as the diameter of the callus divided by the diameter of the original diaphysis (8). The distraction rate was reduced to 0.50 or $0.25 \mathrm{~mm}$ per day if the CDR was $<80 \%$. Distraction was allowed to continue for 16 months. Subsequently, autologous bone grafts were harvested from the ilium and implanted at the non-union site. Bone union was observed for 3 months following the implantation, and the external fixator was removed. Over a follow-up period of 51 months, the patient exhibited no signs of recurrence or metastasis, and at the last follow-up visit in July 2013, the patient's Musculoskeletal Tumor Society functional score (9) was 22. There were no complications during the postoperative course. 

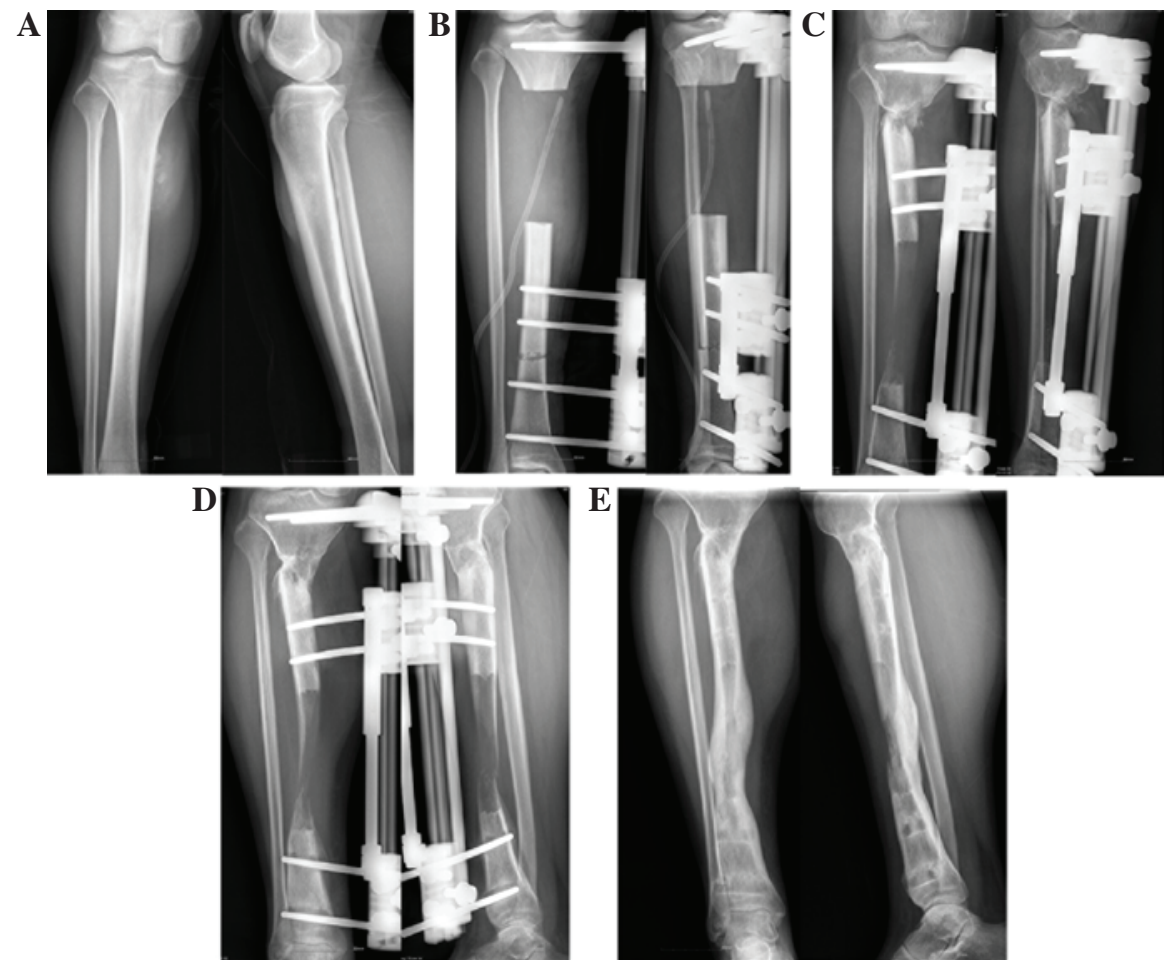

Figure 1. Case 1. Osteosarcoma in the proximal tibial region of a 29-year-old man. (A) Preoperative radiograph showing the lesion. (B) Following resection of the tumor, the tibia was fixed using an Orthofix external fixator. (C) Autologous bone grafts were harvested from the ilium and implanted into the callus 16 months subsequent to initiation of bone transport distraction. (D) Bone union was observed 3 months following bone grafting. (E) Radiograph showing bone consolidation following the removal of the fixator.
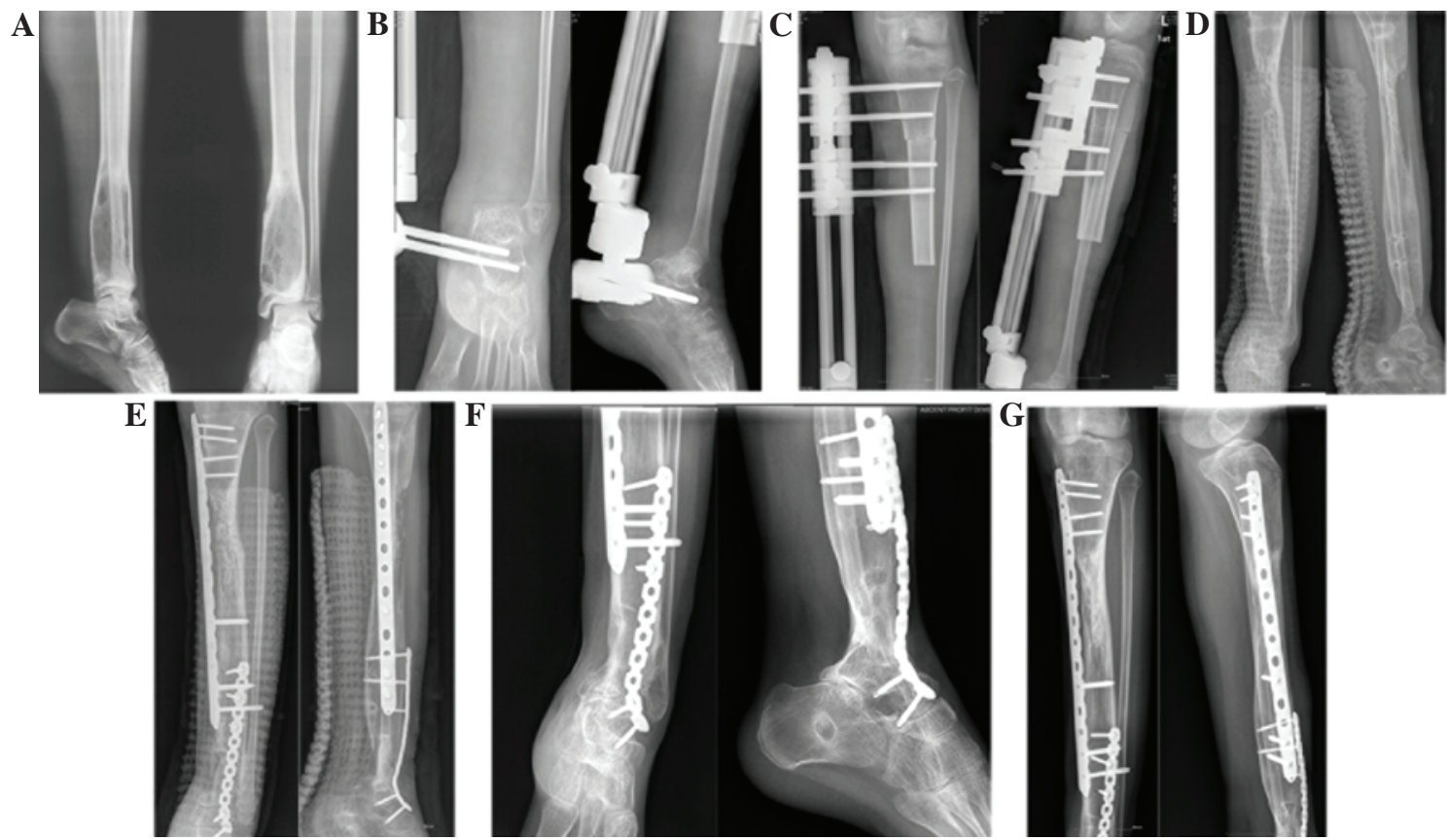

Figure 2. Case 2. Osteosarcoma of the distal tibial region in a 16-year-old girl. (A) Preoperative radiograph showing osteolysis in the distal tibial region. (B and C) The tibial bone defect was fixed using an Orthofix external fixator. (D) Bone consolidation subsequent to 28 months of distraction osteogenesis (E) Fusion of the ankle joint, following autologous grafts from the ilium being implanted into the ankle joint and the callus. An internal fixator was also implanted. ( $\mathrm{F}$ and $\mathrm{G})$ Bone union 3 months following bone grafting.

Case 2. A 16-year-old girl was admitted to the Second Affiliated Hospital in September 2008 with a history of pain in the left ankle, which had been ongoing for 3 months. The lesion was identified at the distal tibial end and no signs of metastasis were noted in the preoperative examination. Pathological examination revealed that the lesion was an osteosarcoma. The patient received preoperative and postoperative chemotherapy each for 2 cycles. Each cycle consisted of methotrexate $\left(8 \mathrm{~g} / \mathrm{m}^{2}\right)$ on 
days 1 and 8 , cisplatin $\left(120 \mathrm{mg} / \mathrm{m}^{2}\right)$ on day 15 and doxorubicin $\left(60 \mathrm{mg} / \mathrm{m}^{2}\right)$ on day 17 . The second cycle of chemotherapy was started after 2 weeks. Following the resection of the tumor, the tibial defect was observed to be $15-\mathrm{cm}$ long and was fixed with an Orthofix Limb Reconstruction System via an external fixator (Orthofix) (Fig. 2). Bone transport was initiated on postoperative day 14 at a distraction rate of $2.0 \times 0.5 \mathrm{~mm}$ per day. As with case 1, the distraction rate was reduced to $0.5 \mathrm{~mm}$ or even $0 \mathrm{~mm}$ per day if the CDR $<80 \%$. Distraction was continued for 28 months. Subsequently, the external fixator was removed, and 4 weeks later autologous bone grafts were harvested from the ilium and implanted at the non-union site, along with an internal fixator. Bone union was observed 3 months later. The patient was followed up for 56 months, at the end of which (in May 2013) the patient exhibited no signs of recurrence or metastasis, and had a Musculoskeletal Tumor Society functional score (9) of 18. There were no complications during the postoperative course.

\section{Discussion}

Several options are now available for the reconstruction of large tibial bone defects following resection of osteosarcoma, including tumor prostheses, allografts, and vascularized, autologous osseous grafts. However, tumor prostheses and allografts are prone to cause infections $(4-6,10)$, and vascularized fibular grafting is a complex procedure that has poor biomechanical strength (11). By contrast, bone transport distraction osteogenesis has been demonstrated to be a low-risk procedure with a beneficial outcome for large bone defects $(7,12)$.

Compared to the other available methods for bone reconstruction, bone transport distraction osteogenesis is a simpler procedure with a shorter operation time and, consequently, lowers the risk of complications. The use of bone transport distraction generally precludes the requirement for internal fixation and prosthesis implantation; therefore, this significantly minimizes the chances of wound infection. The reconstructed bone formed following distraction osteogenesis has good biomechanical performance and sufficient rigidity to allow for the execution of daily activities (13). However, there are certain drawbacks to bone transport distraction. One significant limitation of this method is the long duration required for the completion of distraction, which is a severe test of patient compliance. Furthermore, chemotherapy administered to patients may inhibit the osteogenesis process and reduce the rate of distraction, which may lead to failure of the consolidation of the callus (7). In the two present cases, the distraction rate was adjusted according to the CDR in order to ensure sound formation and consolidation of the callus.

In addition, it is extremely important to select the optimal bone transport rate. Previous studies have demonstrated that bone transport at a rate of $1 \mathrm{~mm}$ per day is optimal for the reconstruction of bone defects caused by trauma $(13,14)$. A low distraction rate of $0.5 \mathrm{~mm}$ per day may lead to premature bone consolidation, whereas a high rate of $2 \mathrm{~mm}$ per day results in the formation of fibrous tissue without osteogenesis. Furthermore, a fixed distraction rate of $1 \mathrm{~mm}$ per day may not be beneficial for patients with osteosarcoma, due to the inhibitory effect of chemotherapy on osteogenesis. CDR is an index that reflects the quality of the callus formation during distraction osteogenesis (8). In the two present cases, the rate of bone transport distraction was reduced to 0.5 or $0.25 \mathrm{~mm}$ per day when the CDR was $<80 \%$. This approach inevitably prolonged the duration of distraction, thereby challenging patient compliance.

Although a few cases (7) have been reported using bone transport distraction osteogenesis for the defects resulting from the removal of bone tumors, the studies have not been concerned with the effects of chemotherapy. The present 2 cases focused on the osteosarcoma, discussing the effects of chemotherapy for bone transport and providing an overview of the whole experience of bone transport distraction osteogenesis. The study was primarily concerned with the effects of chemotherapy for the bone transport distraction osteogenesis of osteosarcoma.

In conclusion, the current study presents two cases in which bone transport was successfully used for the reconstruction of large tibial bone defects following osteosarcoma resection. An important concern associated with this method is the challenge of patient compliance for the entire treatment duration, and measures should be taken to encourage the patient to complete the treatment for successful reconstruction. The current study only presents information on two cases; therefore, a larger case series is required to provide treatment recommendations concerning this type of method for the treatment of tibial bone defects following osteosarcoma resection.

\section{References}

1. Messerschmitt PJ, Garcia RM, Abdul-Karim FW, Greenfield EM and Getty PJ: Osteosarcoma. J Am Acad Orthop Surg 17: 515-527, 2009.

2. Ottaviani $\mathrm{G}$ and Jaffe N: The epidemiology of osteosarcoma. Cancer Treat Res 152: 3-13, 2009.

3. Simon MA: Limb salvage for osteosarcoma in the 1980s. Clin Orthop Relat Res 264-270, 1991.

4. Natarajan MV, Annamalai K, Williams S, Selvaraj R and Rajagopal TS: Limb salvage in distal tibial osteosarcoma using a custom mega prosthesis. Int Orthop 24: 282-284, 2000.

5. Muscolo DL, Ayerza MA, Aponte-Tinao L, Ranalletta M and Abalo E: Intercalary femur and tibia segmental allografts provide an acceptable alternative in reconstructing tumor resections. Clin Orthop Relat Res 97-102, 2004.

6. Ramseier LE, Malinin TI, Temple HT, Mnaymneh WA and Exner GU: Allograft reconstruction for bone sarcoma of the tibia in the growing child. J Bone Joint Surg Br 88: 95-99, 2006.

7. Watanabe K, Tsuchiya H, Yamamoto N, Shirai T, Nishida H, Hayashi K, Takeuchi A, Matsubara H and Nomura I: Over 10-year follow-up of functional outcome in patients with bone tumors reconstructed using distraction osteogenesis. J Orthop Sci 18: 101-109, 2013.

8. Nakamura K, Matsushita T, Mamada K, Okazaki H, Ou W, Okuma Y and Kurokawa T: Changes of callus diameter during axial loading and after fixator removal in leg lengthening. Arch Orthop Trauma Surg 117: 464-467, 1998.

9. Enneking WF, Dunham W, Gehardt MC, Malawer M and Pritchard DJ: A system for the functional evaluation of reconstructive procedures after surgical treatment of tumors of the musculoskeletal system. Clin Orhtop Relat Res: 241-246, 1993.

10. Shekkeris AS, Hanna SA, Sewell MD, Spiegelberg BG, Aston WJ, Blunn GW, Cannon SR and Briggs TW: Endoprosthetic reconstruction of the distal tibia and ankle joint after resection of primary bone tumours. J Bone Joint Surg Br 91: 1378-1382, 2009.

11. Ebeid W, Amin S, Abdelmegid A, Refaat Y and Ghoneimy A: Reconstruction of distal tibial defects following resection of malignant tumours by pedicled vascularised fibular grafts. Acta Orthop Belg 73: 354-359, 2007.

12. Tsuchiya $\mathrm{H}$ and Tomita $\mathrm{K}$ : Distraction osteogenesis for treatment of bone loss in the lower extremity. J Orthop Sci 8: 116-124, 2003.

13. Ilizarov GA: The tension-stress effect on the genesis and growth of tissues: Part II. The influence of the rate and frequency of distraction. Clin Orthop Relat Res 263-285, 1989.

14. Ilizarov GA: Clinical application of the tension-stress effect for limb lengthening. Clin Orthop Relat Res 8-26, 1990. 